\title{
PRODUÇÃO DE SUCO DE ABACAXI OBTIDO A PARTIR DOS RESÍDUOS DA INDÚSTRIA CONSERVEIRA
}

\author{
CAROLINE DELLINGHAUSEN BORGES * \\ JOSIANE FREITAS CHIM * \\ ANGELITA MACHADO LEITÃO * \\ ELISABETE PEREIRA * \\ MÁRCIA DE MELLO LUVIELMO **
}

\begin{abstract}
O presente estudo objetivou o desenvolvimento de suco de abacaxi elaborado a partir dos resíduos das indústrias de conserva da região de Pelotas, Rio Grande do Sul (Brasil). O processamento do suco-base a partir da obtenção das cascas, centros e aparas da fruta consistiu das etapas de branqueamento, prensagem e filtragem. A partir do suco-base foram desenvolvidas formulações com diferentes percentagens de sacarose e adição ou não de corante. A formulação com $7,2 \%$ de sacarose obteve maior índice de aceitação (80,8\%). Conclui-se que é viável a elaboração de suco de abacaxi a partir de resíduos de sua industrialização.
\end{abstract}

PALAVRAS-CHAVE: ABACAXI-RESÍDUOS; SUCO DE ABACAXI.

\section{INTRODUÇÃO}

O abacaxi (Ananas comosus), originário da América do Sul, foi levado para a Europa logo após o descobrimento da América. Seu cultivo disseminou-se por vários países devido grande aceitação do fruto pelos consumidores, justificada pelo atraente sabor e equilíbrio entre ácidos e açúcares (A CULTURA..., 1996; BARTOLOMÉ, RUPÉREZ e FÚSTER, 1995; CANECCHIO, 1973).

Praticamente todos os estados brasileiros cultivam o abacaxi, destacando-se a Paraíba, Bahia e Minas Gerais. A cultivar Smooth

* Químico de Alimentos, Universidade de Pelotas (UFPel), RS.

** Engenheira de Alimentos, Mestre em Nutrição Aplicada a Tecnologia de Alimentos, Universidade Estadual de Campinas (UNICAMP) SP (e-mail: luvielmo@fea.unicamp.br) 
cayenne tem sido a mais utilizada para a industrialização de conservas (A CULTURA..., 1996).

O suco de abacaxi destaca-se entre os sucos de frutas tropicais pelas suas características nutricionais e por ser processado a partir de uma das frutas de maior produção e consumo. Sua industrialização ocorre a partir dos restos procedentes da elaboração do abacaxi enlatado, combinado com o suco que sai do abacaxi quando cortado (A CULTURA..., 1996; BOBBIO e BOBBIO, 1992; CANECCHIO, 1973; GOMES, 1942).

A composição química do fruto depende do estágio de maturação e de fatores agronômicos e ambientais (BARTOLOMÉ, RUPÉREZ e FÚSTER, 1995). Na composição química do abacaxi tem-se 89,9\% de água, $0,3 \%$ de proteínas, $0,5 \%$ de lipídios, 5,8\% de glicídios, 3,2\% de celulose e $0,3 \%$ de sais (FRANCO, 2001). O abacaxi atua como coadjuvante na digestão (CRUESS, 1973; FRANCO e CHALOUB, 1992) em virtude da presença da bromelina. Tal enzima proteolítica, transforma as matérias albuminóides em proteases ou peptanos, tanto em meio ácido como alcalino e neutro.

O fruto apresenta alto teor de açúcares, sendo rico em sais minerais e vitaminas $A, C, B 1$ e B2. Excede a laranja em ferro, contendo quatro vezes mais cálcio do que o trigo integral. Seu consumo é recomendado para crianças, dada a importância desses minerais na formação do sangue e dos ossos (FRANCO e CHALOUB, 1992; FRANCO, 2001).

As cascas e centros do fruto provenientes das indústrias conserveiras podem ser utilizados para fazer calda (empregada nas conservas em lata), para o processamento de vinagre, de geléias, de combustível e para a obtenção de celulose (BOBBIO e BOBBIO, 1992; VARNAM e SUTHERLAND, 1997). Várias pesquisas têm sido desenvolvidas para o aproveitamento dos resíduos do abacaxi. SREENATH, SUDARSHANAKISHNA e SANTHANAM (1994) utilizaram enzimas comerciais (celulase e pectinase) para a extração do suco a partir dos resíduos. Já TANAKA, HILARY e ISHIZAKI (1999) empregaram os resíduos como substrato de baixo custo para produção de etanol por fermentação.

O suco pasteurizado ou fresco é utilizado em refrescos, sorvetes, bombons, confeitos, ácido cítrico, ponche, creme, bala, geléia, licor, 
farinha, vinho e aguardente (CANECCHIO, 1973; FRANCO, 2001; VARNAM e SUTHERLAND, 1997).

A cidade de Pelotas, no Rio Grande do Sul, desfruta de tradição na indústrialização de conservas. Entretanto, essas indústrias geram grande quantidade de resíduos que não são aproveitados (NEVES, 1995). Aproximadamente 65 a $75 \%$ do fruto abacaxi são desperdiçados durante sua industrialização.

O presente estudo objetivou o desenvolvimento de suco de abacaxi elaborado a partir dos resíduos das indústrias de conserva da região de Pelotas, Rio Grande do Sul.

\section{MATERIAL E MÉTODOS}

\subsection{MATERIAL}

Utilizou-se abacaxi (Ananas comosus) da variedade Smooth cayenne, obtido no comércio de Pelotas/RS, selecionado de acordo com seu grau de maturação (coloração amarelo esverdeado), com 12 a 16 Brix e peso entre 2,0 e 2,5 kg. Também foram empregados sacarose (Dolce), corante artificial amarelo damasco (Mix Coralim), corante artificial azul (Heine Essências) e aromatizante de abacaxi (IFF Essências e Fragrâncias).

\subsection{MÉTODOS}

\subsubsection{Pesquisa de Mercado}

Realizou-se pesquisa de mercado com a população do Campus da Universidade Federal de Pelotas, mediante questionário com perguntas objetivas e abordagem direta, a fim de determinar o produto a ser testado (bala, geléia ou suco de abacaxi). Verificou-se também a preferência do consumidor pela cor, aroma, consistência, mistura de sabores e freqüência de consumo para o produto escolhido. Utilizouse amostragem simples e inspeção comum, com nível geral de inspeção dois, que corresponde à amostra de 200 pessoas conforme a Norma ABC-STD - 105D (LOURENÇO FILHO, 1984). 


\subsubsection{Elaboração do produto}

A extração do suco-base de abacaxi foi realizada em pequena escala conforme o fluxograma da Figura 1.

\section{FIGURA 1 - FLUXOGRAMA DE ELABORAÇÃO DO SUCO-BASE DE ABACAXI A PARTIR DE RESÍDUOS DA FRUTA}

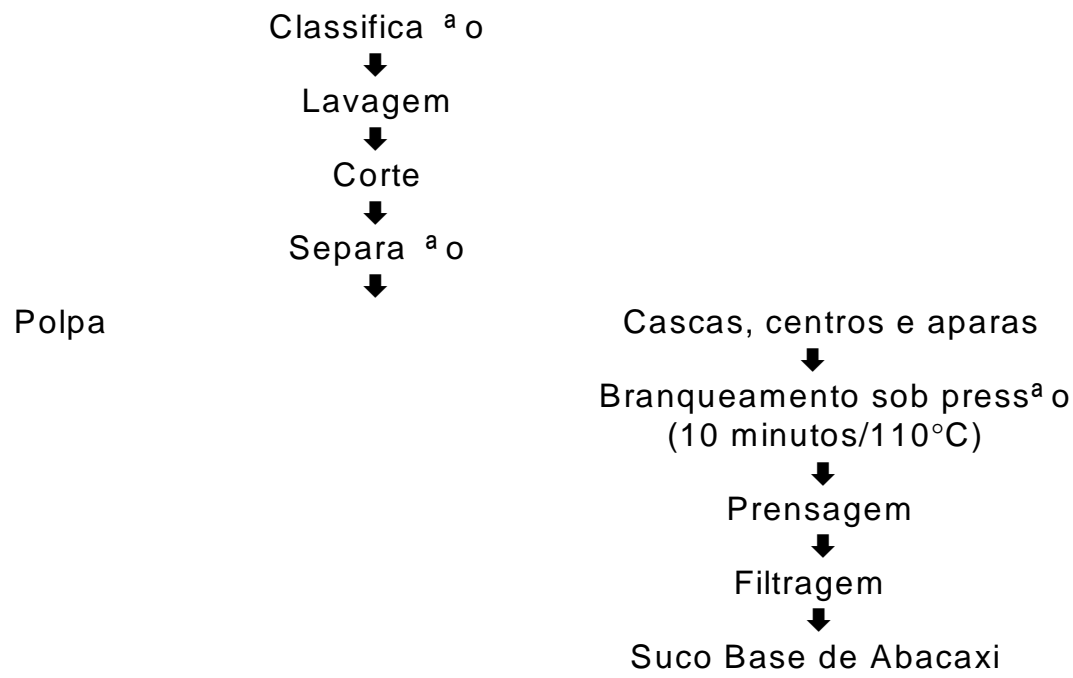

Após a obtenção do suco-base de abacaxi, elaborou-se novo produto de acordo com o resultado da pesquisa de mercado. Foram desenvolvidas quatro formulações, acrescidas de aromatizante e diferentes concentrações de açúcar e de corante (Tabela 1).

\subsubsection{Análise sensorial}

Para a avaliação sensorial dos sucos de abacaxi usou-se o teste de aceitação, com escala hedônica de nove pontos e 100 julgadores nãotreinados (DUTCOSKY, 1996). Os cálculos da análise de variância e do teste de Tukey dos resultados foram realizados com o software Statistica 5.0 (STATSOFT, 1991). 


\section{TABELA 1 - COMPOSIÇÃO PERCENTUAL DAS FORMULAÇÕES DE SUCO DE ABACAXI PROPOSTAS}

\begin{tabular}{|c|c|c|c|c|}
\hline Ingredientes & $\begin{array}{c}\text { Formula }{ }^{\mathrm{a}} \text { o } 1 \\
(\mathrm{~F} 1)\end{array}$ & $\begin{array}{c}\text { Formula }{ }^{a} \circ 2 \\
(\mathrm{~F} 2)\end{array}$ & $\begin{array}{c}\text { Formula }{ }^{a} \text { o } 3 \\
\text { (F3) }\end{array}$ & $\begin{array}{c}\text { Formula }{ }^{a} 04 \\
\text { (F4) }\end{array}$ \\
\hline gua $(\mathrm{mL})$ & 100,0 & 100,0 & 100,0 & 100,0 \\
\hline Abacaxi $(g)$ * & 80,0 & 80,0 & 80,0 & 80,0 \\
\hline A oear $\%(p / v)$ & 5,0 & 7,5 & 5,0 & 7,5 \\
\hline Corante $(\mathrm{ppm})^{* *}$ & 0 & 0 & 0,5 & 0,5 \\
\hline Aromatizante (ppm) & 0,4 & 0,4 & 0,4 & 0,4 \\
\hline
\end{tabular}

* Constituído de cascas $(\mathrm{X})$, centros $(\mathrm{Y})$, aparas $(\mathrm{Z})$ e pequena parte de polpa de abacaxi $(K)$, obedecendo a proporção $X: Y: Z: K=4: 1: 0,5: 0,5$.

** Corante elaborado pela mistura de: $600 \mu \mathrm{L}$ do corante amarelo damasco e $0,01 \mu \mathrm{L}$ do corante azul.

Escolheu-se a formulação que apresentou a maior média e grau de aceitação (superior a 70\%) e efetuou-se modificação no produto com base nos comentários dos julgadores (Tabela 2).

\section{TABELA 2 - COMPOSIÇÃO PERCENTUAL DAS FORMULAÇÕES 5 E 6 DE SUCO DE ABACAXI}

\begin{tabular}{lcc}
\hline Ingredientes & Formula $\stackrel{\text { a }}{0} 5(\mathrm{~F} 5)$ & Formula ${ }^{a}$ o 6 (F6) \\
\hline gua $(\mathrm{mL})$ & 100,0 & 100,0 \\
Abacaxi $(\mathrm{g}) *$ & 80,0 & 80,0 \\
A œar $(\% \mathrm{p} / \mathrm{v})$ & 6,8 & 7,2 \\
Aromatizante $(\mathrm{ppm})$ & 0,4 & 0,4 \\
\hline
\end{tabular}

* Constituído de cascas $(X)$, centros $(Y)$, aparas $(Z)$ e pequena parte de polpa de abacaxi $(K)$ na proporção $X: Y: Z: K=4: 1: 0,5: 0,5$.

\subsubsection{Composição centesimal}

Determinou-se a composição centesimal das duas formulações com maior índice de aceitabilidade (AOAC, 1995), calculando-se o valor calórico total de cada produto (OSBORNE e VOOGT, 1978). 


\section{RESULTADOS E DISCUSSÃO}

\subsection{RESULTADOS DA PESQUISA DE MERCADO}

A pesquisa de mercado revelou preferência dos consumidores pelo suco de abacaxi $(89,0 \%)$ sem mistura com outra fruta $(73,0 \%)$, adocicado $(59,0 \%)$ e com aroma forte $(53,0 \%)$. Também verificou-se preferência pelo suco concentrado $(63,0 \%)$, turvo $(53,3 \%)$ e com baixa acidez $(79,0 \%)$. Desse modo, as formulações de suco foram desenvolvidas visando atender a tais características.

\subsection{RESULTADOS DA ANÁLISE SENSORIAL}

Os resultados do teste de aceitação dos sucos de abacaxi estão apresentados na Tabela 3. O teste de Tukey, ao nível de $5 \%$ de significância, evidenciou que as amostras 2 e 4 com 7,5\% de sacarose diferiram significativamente da 1 e da 3 (com 5,0\% de sacarose).

\section{TABELA 3 - MÉDIAS E DESVIOS-PADRÕES OBTIDOS NO TESTE DE ACEITAÇÃO PARA AS FORMULAÇÕES 1, 2, 3 e 4}

\begin{tabular}{|c|c|c|}
\hline Amostras & MØdias & Desvio Padr ${ }^{a} 0$ \\
\hline F1 (5,0\% SC) & $6,13 \mathrm{~b}$ & 1,56 \\
\hline $\mathrm{F} 2(7,5 \% \mathrm{SC})$ & $7,11 \mathrm{a}$ & 1,37 \\
\hline F $3(5,0 \%$ CC $)$ & $5,84 \mathrm{~b}$ & 1,81 \\
\hline F $4(7,5 \%$ CC $)$ & $7,22 \mathrm{a}$ & 1,39 \\
\hline
\end{tabular}

Os sucos com $7,5 \%$ de sacarose obtiveram maiores valores de aceitabilidade, sendo $80,2 \%$ para a amostra com corante e $79,0 \%$ para a sem corante (Figura 2). A presença do corante não influenciou significativamente $(p<0,05)$ a aceitação dos sucos. 
Foram elaboradas duas formulações a partir da amostra 4 que obteve maior índice de aceitabilidade, considerando a sugestão de redução da sacarose (Tabela 4).

\section{FIGURA 2 - GRÁFICO DOS ÍNDICES DE ACEITABILIDADE DAS FORMULAÇÕES F1, F2, F3, F4, F5 e F6}

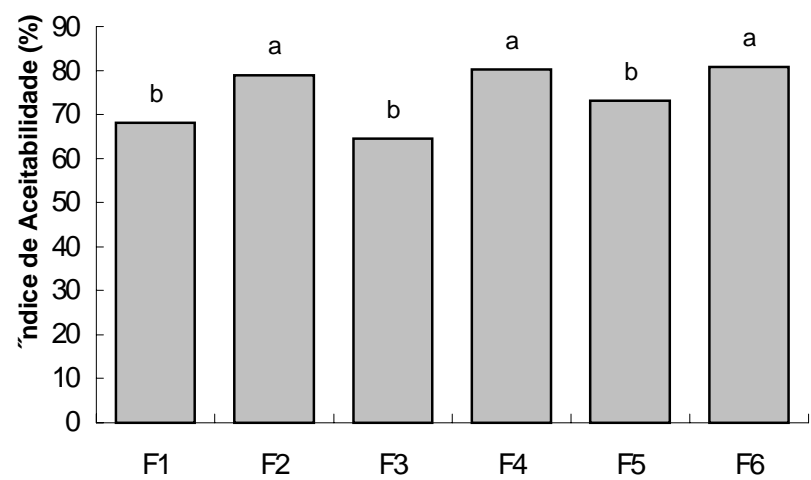

\section{TABELA 4 - MÉDIAS E DESVIOS-PADRÕES OBTIDOS NO TESTE DE ACEITAÇÃO PARA AS FORMULAÇÕES 5 E 6}

\begin{tabular}{lcc}
\hline Amostras & MØdias & Desvio Padr ${ }^{\mathrm{a}}$ o \\
\hline F5 (6,8\% SC) & $6,58 \mathrm{~b}$ & 1,42 \\
F6 $(7,2 \%$ SC) & $7,28 \mathrm{a}$ & 1,31 \\
\hline
\end{tabular}

$\mathrm{SC}=$ Sem corante.

Médias seguidas por letras iguais não diferem entre si pelo teste de Tukey, ao nível de $5 \%$ de significância.

Verificou-se que ambas as amostras apresentaram índice de aceitabilidade superior a 70\%, com destaque para a formulação 6 (7,2 g de açúcar/100 $\mathrm{mL}$ de açúcar). A redução de 7,5\% para 7,2\% de sacarose mostrou-se interessante por possibilitar diminuição no custo final do produto, embora não tenha sido verificada diferença significativa $(p<0,05)$ entre as amostras 4 e 6. 


\subsection{RESULTADOS DAS ANÁLISES FÍSICO-QUÍMICAS}

Os resultados das análises físico-químicas do suco de abacaxi estão apresentados na Tabela 5.

\section{TABELA 5- COMPOSIÇÃO CENTESIMAL DO SUCO DESENVOLVIDO (F6)}

\begin{tabular}{lc}
\hline \multicolumn{1}{c}{ Composi a o centesimal } & Suco F6 \\
\hline Prote nas & 0,25 \\
Lip dios & 0,90 \\
Carboidratos & 8,40 \\
Fibras & 0,05 \\
Cinzas & 0,01 \\
Umidade & 90,40 \\
Calorias (kcal) & 39,0 \\
\hline
\end{tabular}

A composição centesimal do suco obtido enquadrou-se nos padrões fixados para suco de abacaxi de acordo com o Ministério da Agricultura, Pecuária e Abastecimento (BRASIL, 2003).

O suco desenvolvido apresentou composição similar à de outras marcas de suco de abacaxi oferecidas no mercado, diferindo apenas no conteúdo de carboidratos e no valor calórico (69\% menos carboidratos e $64 \%$ menos calorias). Em função dessa observação e dos resultados obtidos cogitou-se que os produtos oferecidos no mercado não necessitam de tanta sacarose para atingir alto índice de aceitação por parte dos consumidores.

\section{CONCLUSÃO}

O suco de abacaxi desenvolvido a partir de resíduos da industrialização apresentou $80,8 \%$ de aceitabilidade. Além de alternativa econômica e ecologicamente viável, a produção desse suco possibilita a transformação de resíduos em fonte lucrativa. 


\section{Abstract}

\section{PRODUCTION OF PINEAPPLE JUICE OBTAINED FROM THE RESIDUES OF THE CONSERVE INDUSTRY}

The aim of the present study was the development of pineapple juice elaborated from the residues of the conserve industries of Pelotas region, Rio Grande do Sul (Brazil). The base-juice processing from the obtention of husks, centers and shavings of the fruit consisted of the stages of bleaching, pressing and filtering. From the base-juice formulations were developed with different percentages of saccharose and the addition or not of colorants. The formulation with $7.2 \%$ of saccharose obtained higher acceptation index (80.8\%). It was concluded that the elaboration of pineapple juice from its industrialization residues is viable.

KEY-WORDS: PINEAPPLE-RESIDUES; PINEAPPLE JUICE.

\section{REFERÊNCIAS}

1 AOAC. Association of Official Analytical Chemists. Official methods of analysis of AOAC international. $16^{\text {th }}$ ed. Washington, 1995.

2 A CULTURA do abacaxi: perspectivas, tecnologias e viabilidade. Rio de Janeiro: Pesagro, 1996. p. 27.

3 BARTOlOMÉ, A. P.; RUPÉREZ, P.; FÚSTER, C. Pineapple fruit: morphological characteristics, chemical composition and sensory analysis of red spanish and Smooth cayenne cultivars. Food Chemistry, v. 53, p. 75-79, 1995.

4 BOBBIO, F.O.; BOBBIO, P.A. Introdução à química de alimentos. 2.ed. São Paulo: Varela, 1992. p. 223.

5 BRASIL. Ministério da Agricultura, Pecuária e Abastecimento. Instrução Normativa n. 12 de 4 de setembro de 2003. Regulamento técnico para fixação dos padrões de identidade e qualidade gerais para suco tropical. Diário Oficial [da] República Federativa do Brasil, Brasília, 09 de setembro de 2003. Seção 1, p. 2.

6 CANECCHIO FILHO, V. Indústrias rurais. 2.ed. São Paulo: Instituto Campineiro de Ensino Agrícola, 1973. v.12.

7 CRUESS, W.V. Produtos industriais de frutas e hortaliças. São Paulo: Edgard Blücher, 1973. v. 2.

8 DUTCOSKY, S.D. Análise sensorial de alimentos. Curitiba: Champagnat, 1996. p. 84-85.

9 FRANCO, G.; CHALOUB, S. R. Dietas e receitas: valores calóricos e propriedades gerais dos alimentos. 3.ed. São Paulo: Atheneu, 1992. p. 395. 
10 FRANCO, G. Tabela de composição química dos alimentos. 9.ed. São Paulo: Atheneu, 2001. p. 230.

11 GOMES, P. Fruticultura brasileira. 12.ed. São Paulo: Nobel, 1942. p. 446.

12 LOURENÇO FILHO, R. C. B. Controle estatístico de qualidade. São Paulo/ Rio de Janeiro: Livros Técnicos e Científicos, 1984. p. 204-205.

13 NEVES, N. de A. Preparo de alimentos nutritivos e saborosos. 4.ed. Pelotas: Universidade Federal de Pelotas, 1995. 250 p.

14 OSBORNE, D. R.; VOOGT, P. Análisis de los nutrientes de los alimentos. Zaragoza: Acribia, 1978. p. 290.

15 SREENATH, H. K.; SUDARSHANAKISHNA, K.; SANTHANAM, K. Improvement of juice recovery from pineapple pulp/residue using cellulases and pectinases. Journal of Fermentation and Bioengineering, v. 78, n.61, p. 486-488, 1994.

16 STATSOFT. Statistica. Tulsa: Statsoft, 1991. v.1, p. 935.

17 TANAKA, K.; HILARY, Z. D.; ISHIZAKI, A. Investigation of utility of pineapple juice and pineapple waste material as low-cost substrate for ethanol fermentation by Zymomonas mobilis. Journal of Bioscience and Bioengineering, v. 87, p. 642-646, 1999.

18 VARNAM, A. H.; SUTHERLAND, J. P. Bebidas, tecnologia, química y microbiologíca. Zaragoza: Acríbia, 1997. p. 487. 